\title{
A POWER-FREE LIQUID DRIVEN METHOD FOR MICRO MIXING APPLICATION
}

\author{
${ }^{1}$ Chien-Fu Chen, ${ }^{2}$ Shih-Chi Kuo, ${ }^{3} \mathrm{Chin}-\mathrm{Chou} \mathrm{Chu}$, and ${ }^{4} \mathrm{Fan}-\mathrm{Gang}$ Tseng, \\ ${ }^{1,3}$ Institute of Applied Mechanics, National Taiwan University, Taiwan \\ ${ }^{2,4}$ Engineering and System Science Dept., National Tsing Hua University, Taiwan
}

\begin{abstract}
This paper proposes a novel method to perform micro mixing without any active devices such as pumps, valves, or external energies like electrostatic, or magnetic fields, which may have negative interactions with working fluids. In this novel mixing device, surface tension force from the working fluid is the only energy resource employed passively to transport, merge, mix, and stop liquid automatically by the design of channel structure and surface properties. The fluidic channel was designed without sidewall and confined with only the bottom hydrophilic and top hydrophobic surface for later-on mixing process among channels, and with spiral channel shape for saving space and shortening channel distance to one another. Fabrication has been completed successfully, and the testing result demonstrated effective fluid flow in spiral channel by surface tension once the liquid is dropped on the entrance, as well as the mixing between two different liquids without extra actuation. This device can be applied to power-free $\mu$ TAS or lab-on a-chip system.
\end{abstract}

\section{INTRODUCTION}

Mixing in micro scale is an important yet challenge issue for the application of $\mu$ TAS (Micro Total Analysis System). No matter mixing is either performed by passive $[1,2]$ or active ways [3,4], external energy sources, such as pumps, compressors, electrical or magnetic energies are usually required for fluid manipulation.

There have been many actuation means to drive fluids in micro system, for example, by providing pressure gradient [1-4], using thermal energies $[5,6]$, or employing electrostatic force $[7,8]$. However, most of them required either actuators like pumps or valves, or external energies such as electrostatic or magnetic fields to drive the fluidic inside micro channel, and most of time they need a large outside energy sources to support the desiring actuation, which greatly limit the capability of system miniaturization and integration.

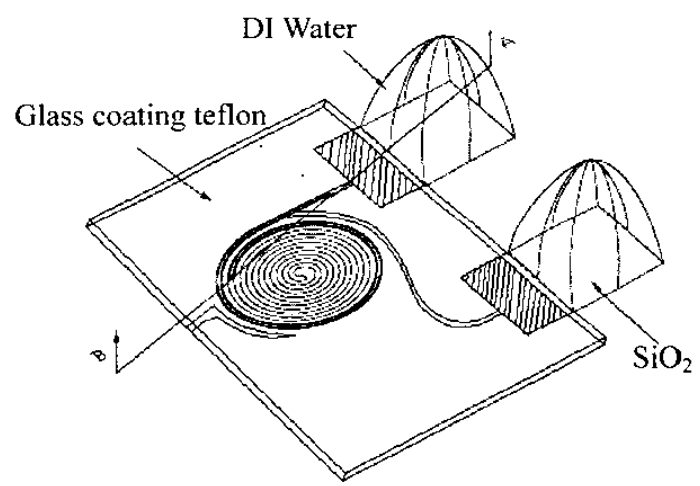

Fig 1. The microstructure of micromixer.

As a result, a power-free device is proposed in the present study employing only surface 
tension energy defined by channel shape and surface properties of the flow passages, to manipulate dual fluids for mixing purpose.

\section{DESIGN CONCEPTS}

The design of the mixing device, as shown in Fig. 1, comprises a double fluidic entrance defined by only the bottom substrate with hydrophilic pattern, and a spiral channel confined by the bottom hydrophilic pattern and a top hydrophobic wall employed to separate fluid in different channel temporarily, as the cross section shown in Fig. 2a. Once fluids are pipetted into the entrances, the pressure pushing-force established by the liquid puddle and the surface tension pulling-force generated by the liquid meniscus close to the hydrophilic side drag the fluids into the sidewall-free fluid passage, as shown in Fig. 2b. The fluid will be stopped at the end of the spiral channel. When two fluids are introduced onto either liquid entrance, the two fluids will be driven automatically by surface tension into the spiral channel and meet with each other for premixing.
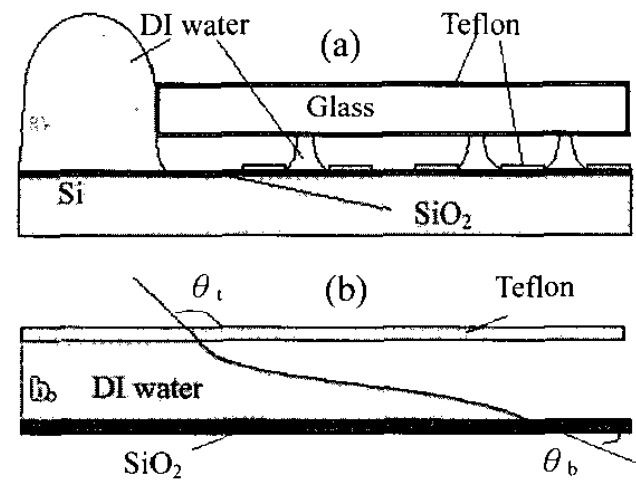

Fig 2. Cross-section of liquid driving.

\section{FABRICATION}

Fabrication process, as shown in Fig. 3, is performed by patterning Teflon on silicon wafer with $\mathrm{SiO}_{2}$ coating as the bottom hydrophilic channel guide, and the cover glass slide coated with Teflon as a hydrophobic liquid separation layer.

Fabrication starts from the deposition of PECVD silicon oxide on silicon substrate (Fig. 3a). 1\% liquid Teflon (Dupont, USA) is then spun on the oxide surface and patterned to form hydrophobic area (Fig. 3b). The process followed by Photoresist (SJR5740) patterning on silicon substrate as spacers for channel height definition (Fig. $3 \mathrm{c}$ ), and Teflon coating on both side of a glass substrate (Fig. 3d) as the channel cover. Finally, The top and bottom substrates are bonded by photoresist with desired clearance, as shown in Fig. $3 \mathrm{e}$.
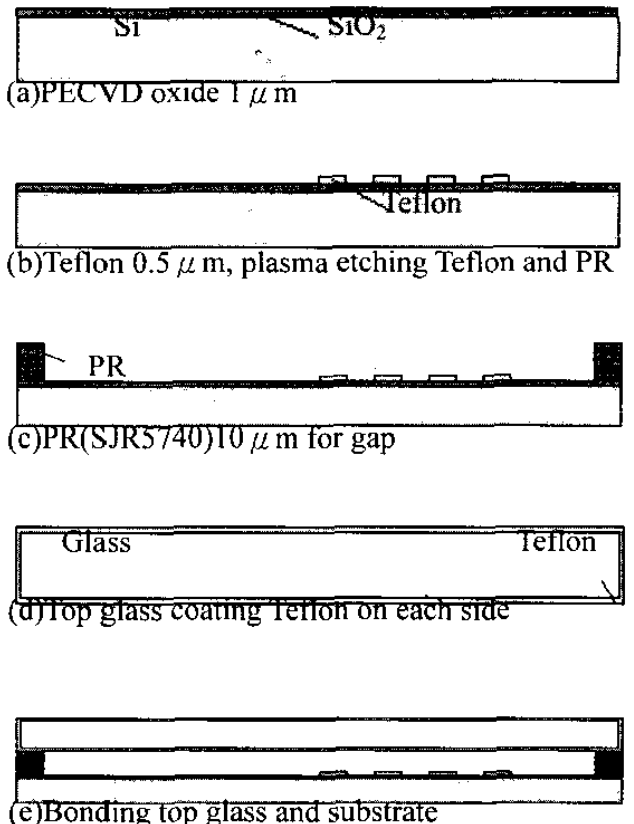

Fig 3. fabrication process. 
The fabricated mixer chip is shown in Fig. 4.

The plasma treated bottom surface showed hydrophilic in the channel region with $\mathrm{SiO}_{2}$ coating, but hydrophobic at the rest area with Teflon and resist coating. Thus, liquid can stay inside hydrophilic channel area once dropped in.

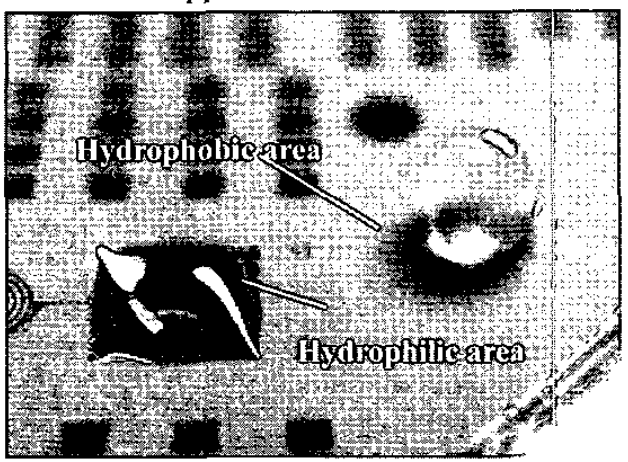

Fig 4. The physical phenomenon after plasma etching.

\section{EXPERIMENT RESULTS}

To demonstrate the functionality of the fluid driving device, two experiments have been performed.

Firstly the fill-in process for one liquid inside the top-bottom confined spiral channel was visualized and analyzed. In Fig. 5, DI water was introduced into the entrance of the micro channel of $100 \mu \mathrm{m}$

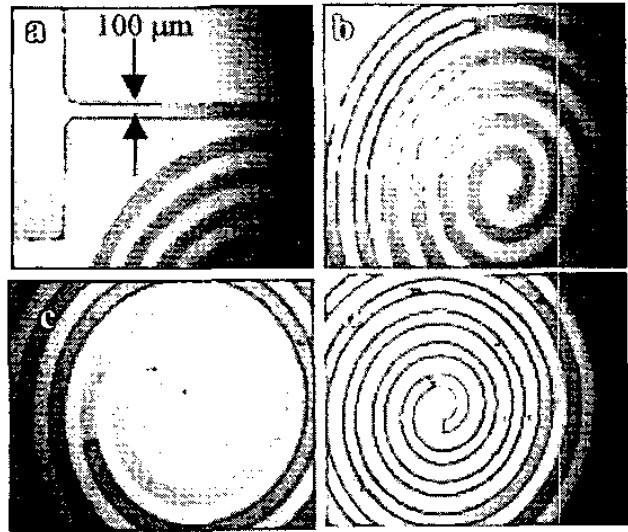

Fig 5 Testing of liquid driving wide and $3 \mathrm{~cm}$ long, and the liquid can be brought unto the end of the, channel automatically without any other driving means than surface tension and hydraulic pressure. The velocity of the meniscus varied from $0.5 \mathrm{~cm} / \mathrm{s}$ to $1.7 \mathrm{~cm} / \mathrm{s}$ due to the drag force increased by the increasing of liquid-solid interface. The filling process was completed within 30 seconds.

Secondly, premixing of two solutions was performed in the spiral channel with dual liquid entrance. Red and green Fluorescent spheres (R900,Duke Scientific Corp.) were added into DI water to form. working fluids. The concentration is $1 \% \mathrm{w} / \mathrm{w}$, and diameter of the spheres is $0.93 \mu \mathrm{m}$. The mixing process was also visualized by CCD camera under microscope, and the mixing process is shown in Fig. 6. DI water with two different contents were pipetted into each entrance (Fig. 6 a-b), liquids meet with each other and merge (Fig. $6 \mathrm{c}-\mathrm{d})$, the merged liquid flows forward for further mixing process (Fig. 6 e-f), and

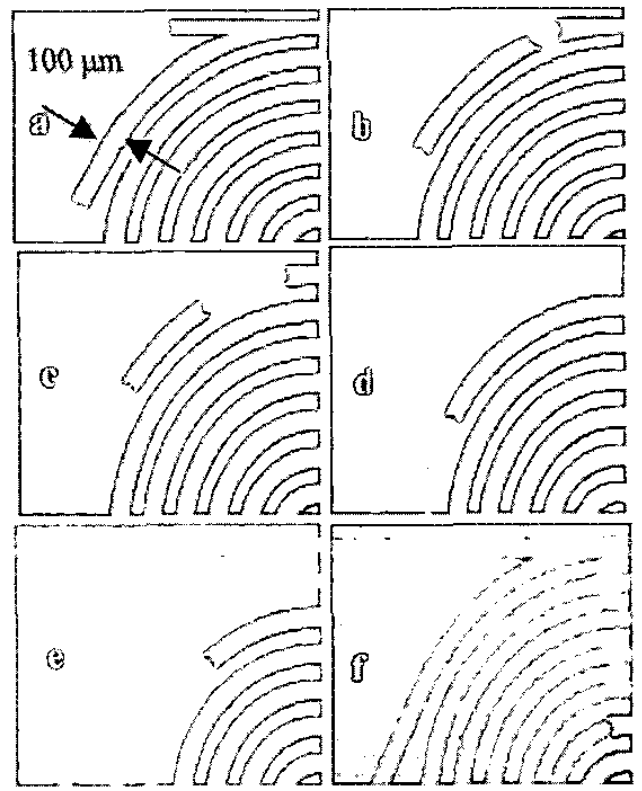

Fig 6. Testing of liquid 
finally stop at the end of spiral channel. Liquid can be cut at the entrance point to precisely define the required mixing amount. This mixer provides the premixing function and is one part of a mciro total analysis system.

\section{CONCLUSIONS}

A power-free liquid driven method by surface tension and hydraulic pressure has been proposed in this paper for premixing application. Fabrication has been fully completed, and the designed hydrophilic channel area, hydrophobic spacer area, has also been demonstrated and showed feasibility for liquid confinement. Test results demonstrated fast liquid driven-in within 12 seconds on a $3 \mathrm{~cm}$ long spiral channel, and premixing process by two DI water-fluorescent sphere mixtures. This device can be applied to power-free micro total analysis system.

\section{ACKNOWLEDGEMENTS}

The work was supported by the National Science Council of Republic of China under the grant NSC-90-2212-E-002-204-.

\section{REFERENCES}

[1] Schwesinger, N., Frank, T., and Wurmus, H.,"A modular microfluid system with an integrated micromixer", J. Micromech. Microengr., Vol.6, pp. 99-102, 1996.

[2] Branebjerg, J.; Gravesen, P.; Krog, J.P.; Nielsen, C.R.,"Fast mixing by lamination"
1996, Proceedings IEEE MEMS '96, pp. 441 -446. 1996.

[3] Suzuki, H.; C.M. Ho,"A magnetic force driven chaotic micromixer", Proceedings IEEE MEMS'02, pp. 40-43, 2002.

[4] Deval, J. ;Tabeling, P.; C.M. Ho, "A dielectrophoretic chaotic mixer Deval" Proceedings IEEE MEMS'02, pp. 36-39, 2002.

[5] Elwenspoek, M., Lammerink, T. S. J., Mijake, and Fluitman, L. H.," A simple micropump employing dynamic pressure valves made in silicon", J. Micromech. Microengr. 4, 227, 1994.

[6] Thomas K.Jun and C.J.Kim, "Valveless pumping using traversing vapor bubbles in microchannels", Journal of Applied Physics, Vol 83, No.11, 1 June 1998.

[7] Masao Washizu, "Electrostatic Actuation of Liquid Droplets for Microreactor Application", IEEE TRANSACTIONS ON INDUSTRY, Vol.34, NO 4, pp.732-737, JULY/AUGUST 1998.

[8] Junghoon Lee and C.J. Kim," Liquid Micromotor Driven By Continuous Electrowetting", Proc. 1998 IEEE MEMS Conference, pp.538-543, Heidelberg, Germany, 1998. 